\begin{tabular}{|c|c|}
\hline Title & A symmetric addition of arylboronic acids to gly oxylate catalyzed by a rutheniumMe-BIPAM complex \\
\hline Author(s) & Y amamoto, Y asunori; Shirai, Tomohiko; Miy aura, Norio \\
\hline Citation & $\begin{array}{l}\text { Chemical Communications, 48(22), 2803-2805 } \\
\text { https://doi.org/10.1039/c2cc17339e }\end{array}$ \\
\hline Issue Date & 2012-03-14 \\
\hline Doc URL & http:/hdl.handle.net/2115/51152 \\
\hline Rights & Chem. Commun., 2012, 48(22), 2803-2805. - Reproduced by permission of The Royal Society of Chemistry (RSC) \\
\hline Type & article (author version) \\
\hline File Information & CC48-22_2803-2805.pdf \\
\hline
\end{tabular}

Instructions for use 


\title{
Asymmetric addition of arylboronic acids to glyoxylate catalyzed by ruthenium/Me-BIPAM complex
}

\author{
Yasunori Yamamoto, * Tomohiko Shirai and Norio Miyaura \\ Received (in $X X X, X X X) X t h X X X X X X X X X 20 X X$, Accepted Xth $X X X X X X X X X 20 X X$ \\ ${ }_{5}$ DOI: 10.1039/b000000x
}

The enantioselective synthesis of $\alpha$-hydroxy esters by ruthenium-catalyzed 1,2-addition of arylboronic acids to tertbutyl glyoxylate is described. The use of $\operatorname{RuCl}_{2}\left(\mathrm{PPh}_{3}\right)_{3}$ with $(R, R)$-Me-BIPAM gave optically active mandelic acids of up 10 to $99 \%$ ee. Addition of a fluoride salt such as potassium fluoride $\mathrm{KF}$ or cesium fluoride $\mathrm{CsF}$ was effective for achieving high enantioselectivities.

Optically active mandelic acid derivatives are very important chiral building blocks for the synthesis of various bioactive 15 products. ${ }^{1}$ Several synthetic methods for these compounds have been developed, including enzymatic methods, ${ }^{2}$ Cannizzaro reaction, ${ }^{3}$ enantioselective reduction ${ }^{4}$ and hydrogenation ${ }^{5}$ of $\alpha$ ketoester, Friedel-Crafts reaction ${ }^{6,7}$ and kinetic resolution. ${ }^{8}$ Recently, catalytic enantioselective arylation of glyoxylate has 20 been reported. ${ }^{9}$ We have already reported a new catalytic cycle starting from transmetalation to give an organorhodium(I), ${ }^{10}$ palladium(II) ${ }^{11}$ or -ruthenium(II) ${ }^{12}$ intermediate for 1,4 -addition of organoboronic acids to electron-deficient alkenes and arylation of the carbon-heteroatom double bond of aldehydes or $N$-sulfonyl 25 imines. In addition, we have developed new bidentate chiral phosphoramidites (Me-BIPAM, ${ }^{12-14} N$-Me-BIPAM ${ }^{15}$ ) on the basis of linked-BINOL for enantioselective 1,4-addition of arylboronic acids to enones, ${ }^{13}$ arylation of aldimines ${ }^{15}$ and hydrogenation of $\gamma$-dehydroamino esters ${ }^{14}$ with rhodium catalysts. These ligands 30 were also found to be highly efficient for ruthenium-catalyzed enantioselective arylation of aldehydes and $\alpha$-ketoesters. ${ }^{12}$ Herein, we report asymmetric arylation of glyoxylate (1) with arylboronic acids (2) catalyzed by a chiral ruthenium complex, generated in situ from $\mathrm{RuCl}_{2}\left(\mathrm{PPh}_{3}\right)_{3}$ and $(R, R)$-Me-BIPAM (Scheme 1).

35 Our initial investigation began by screening catalysts to evaluate their ability to promote the enantioselective arylation of ethyl glyoxylate with phenylboronic acid. As shown in Table 1, since the rhodium complex was inefficient, the use of ruthenium as the central metal is critical for achieving enantioselectivities. ${ }_{40} \mathrm{We}$ have already reported $\left[\mathrm{RuCl}_{2}(p \text {-cymene })\right]_{2} /(R, R)$-Me-BIPAM complex-catalyzed highly enantioselective arylation of

Division of Chemical Process Engineering, Graduate School of Engineering, Hokkaido University, kita 13, nishi 8, Kita-ku, Sapporo 06045 8628, Japan. E-mail: yasuyama@eng.hokudai.ac.jp; Fax\&Tel: +81-11706-6560

†Electronic Supplementary Information (ESI) available: See DOI: 10.1039/b000000x/

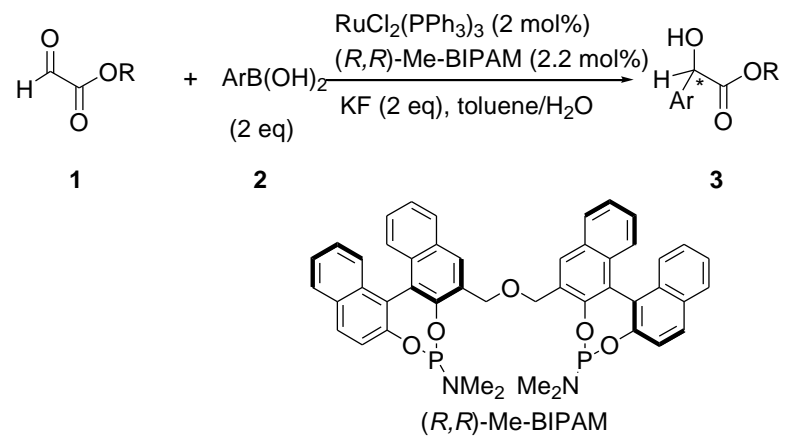

50 Scheme 1 Asymmetric addition of arylboronic acids to glyoxylate.

aldehydes. ${ }^{12}$ As an initial experiment under similar conditions, the reaction in the presence of $2 \mathrm{~mol} \%$ of $\left[\mathrm{RuCl}_{2}(p \text {-cymene })\right]_{2}$ and $2.2 \mathrm{~mol} \%$ of $(R, R)$-Me-BIPAM resulted in $25 \%$ yield and $2 \%$ ee (entry 2). Several combinations of ruthenium(II) precursors, 55 bases and ester alkyl groups revealed the high efficiency of $\mathrm{RuCl}_{2}\left(\mathrm{PPh}_{3}\right)_{3}$ and $\mathrm{KF}$ in toluene for the addition of $\mathrm{PhB}(\mathrm{OH})_{2}$ to $t$-butyl glyoxylate (entry 7). Since hydrolysis of the ester is suppressed, the yield of the product was dependent on the bulkiness of the ester moiety of the substrate, as ethyl (59\% yield), $60 i$-propyl (79\% yield), and $t$-butyl (90\% yield).

Table 1 Reaction conditions. ${ }^{a}$

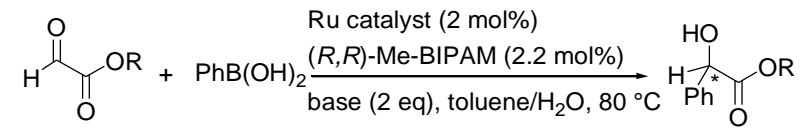

\begin{tabular}{|c|c|c|c|c|c|c|}
\hline entry & $\mathrm{R}=$ & catalyst & base & solvent & yield $/ \%$ & ee $/ \%$ \\
\hline 1 & Et & $\mathrm{Rh}(\mathrm{nbd})_{2} \mathrm{BF}_{4}$ & KF & toluene & 36 & 4 \\
\hline 2 & Et & {$\left[\mathrm{RuCl}_{2}(p \text {-cymene })\right]_{2}$} & KF & toluene & 25 & 2 \\
\hline 3 & Et & $\mathrm{RuCl}_{2}(\mathrm{nbd})(\mathrm{MeCN})_{2}$ & KF & toluene & 39 & 84 \\
\hline 4 & Et & $\operatorname{RuCl}(p$-cymene $)(\mathrm{MeCN})_{2} \mathrm{PF}_{6}$ & KF & toluene & 17 & 0 \\
\hline 5 & Et & $\mathrm{RuCl}_{2}\left(\mathrm{PPh}_{3}\right)_{3}$ & KF & toluene & 59 & 93 \\
\hline 6 & $i$-Pr & $\mathrm{RuCl}_{2}\left(\mathrm{PPh}_{3}\right)_{3}$ & KF & toluene & 79 & 94 \\
\hline 7 & $t-\mathrm{Bu}$ & $\mathrm{RuCl}_{2}\left(\mathrm{PPh}_{3}\right)_{3}$ & KF & toluene & 90 & 96 \\
\hline 8 & $t-\mathrm{Bu}$ & $\mathrm{RuCl}_{2}\left(\mathrm{PPh}_{3}\right)_{3}$ & $\mathrm{CsF}$ & toluene & 61 & 92 \\
\hline 9 & $t-\mathrm{Bu}$ & $\mathrm{RuCl}_{2}\left(\mathrm{PPh}_{3}\right)_{3}$ & $\mathrm{~K}_{2} \mathrm{CO}_{3}$ & toluene & 52 & 55 \\
\hline 10 & $t-\mathrm{Bu}$ & $\mathrm{RuCl}_{2}\left(\mathrm{PPh}_{3}\right)_{3}$ & $\mathrm{~K}_{3} \mathrm{PO}_{4}$ & toluene & 26 & 71 \\
\hline 11 & $t-\mathrm{Bu}$ & $\mathrm{RuCl}_{2}\left(\mathrm{PPh}_{3}\right)_{3}$ & $\mathrm{KF}$ & toluene $^{b}$ & 33 & 91 \\
\hline 12 & $t-\mathrm{Bu}$ & $\mathrm{RuCl}_{2}\left(\mathrm{PPh}_{3}\right)_{3}$ & $\mathrm{KF}$ & $\mathrm{CH}_{2} \mathrm{Cl}_{2}$ & 23 & 13 \\
\hline 13 & $t-\mathrm{Bu}$ & $\mathrm{RuCl}_{2}\left(\mathrm{PPh}_{3}\right)_{3}$ & $\mathrm{KF}$ & THF & trace & - \\
\hline
\end{tabular}

a Reaction conditions: A mixture of glyoxylates $(0.5 \mathrm{mmol})$, phenylboronic acid $(1.0 \mathrm{mmol})$, base $(1.0 \mathrm{mmol}), \mathrm{Ru}$ catalyst $(2 \mathrm{~mol} \%)$, 65 and $(R, R)$-Me-BIPAM $(2.2 \mathrm{~mol} \%)$ in toluene $(3 \mathrm{ml})$ and $\mathrm{H}_{2} \mathrm{O}(0.3 \mathrm{ml})$ was stirred at $80{ }^{\circ} \mathrm{C}$ for 16 h. ${ }^{\mathrm{b}}$ toluene $/ \mathrm{H}_{2} \mathrm{O}=5 / 1$ 
On the other hand, other bases such as $\mathrm{K}_{2} \mathrm{CO}_{3}$ and $\mathrm{K}_{3} \mathrm{PO}_{4}$ previously used for addition to aldehydes were not effective (entries 9 and 10). It is interesting that increase of water resulted in lower yield (entry 11). The reaction also failed when other 5 solvents such as $\mathrm{CH}_{2} \mathrm{Cl}_{2}$ and THF were used. Finally, the desired product was selectively afforded in $90 \%$ yield and $96 \%$ ee when the reaction was carried out at $80{ }^{\circ} \mathrm{C}$ in toluene/ $\mathrm{H}_{2} \mathrm{O}(10 / 1)$ in the presence of a $\mathrm{RuCl}_{2}\left(\mathrm{PPh}_{3}\right)_{3} /(R, R)$-Me-BIPAM catalyst $(2 / 2.2$ $\mathrm{mol} \%)$. Results of arylation of tert-butyl glyoxylate with 10 representative arylboronic acids are summarized in Table 2. Representative para- and meta-substituted arylboronic acids with electron-donating or electron-withdrawing substituents afforded mandelic acid derivatives in good yields with high enantioselectivities in the range of $76-99 \%$ ee. 3-

${ }_{15}$ Chlorophenylboronic acid resulted in $16 \%$ ee when $\mathrm{RuCl}_{2}\left(\mathrm{PPh}_{3}\right)_{3}$ was used as a catalyst precursor, and the ee was increased to $88 \%$ ee with 54\% yield to reduce the steric hindrance than that of $\mathrm{PPh}_{3}$ when $\mathrm{PMePh}_{2}$ was added as a ligand (entries 8 and 9).

Table 2 Arylation of tert-butyl glyoxylate. ${ }^{a}$

entry

${ }^{\text {a }}$ Reaction conditions: A mixture of glyoxylates $(0.5 \mathrm{mmol})$, aryl boronic acid (1.0 mmol), $\mathrm{KF}(1.0 \mathrm{mmol}), \mathrm{RuCl}_{2}\left(\mathrm{PPh}_{3}\right)_{3}(2 \mathrm{~mol} \%)$, and $(R, R)-\mathrm{Me}-$ BIPAM $(2.2 \mathrm{~mol} \%)$ in toluene $(3 \mathrm{ml})$ and $\mathrm{H}_{2} \mathrm{O}(0.3 \mathrm{ml})$ was stirred at 80 ${ }^{\circ} \mathrm{C}$ for $16 \mathrm{~h} .{ }^{\mathrm{b}}$ The letter within the parentheses indicates the absolute 25 configuration of the chiral center within the product. ${ }^{\mathrm{c}} \mathrm{PMePh}_{2}(2.2$ mol\%) was added
We propose a possible catalytic cycle of this reaction (Figure 1). The reaction may proceed through transmetalation of $\left[\operatorname{ArBF}_{n}(\mathrm{OH})_{(3-n)}\right] \mathrm{K}$ to a ruthenium(II) complex giving $\mathrm{Ar}-[\mathrm{Ru}]$. 30 Insertion of the $\mathrm{C}-\mathrm{O}$ double bond into the $\mathrm{C}-\mathrm{Ru}$ bond, giving $[\mathrm{Ru}]\left(\mathrm{OCH}(\mathrm{Ar}) \mathrm{CO}_{2}{ }^{t} \mathrm{Bu}\right) \mathrm{Cl}$, can then undergo hydrolysis. ${ }^{12}$

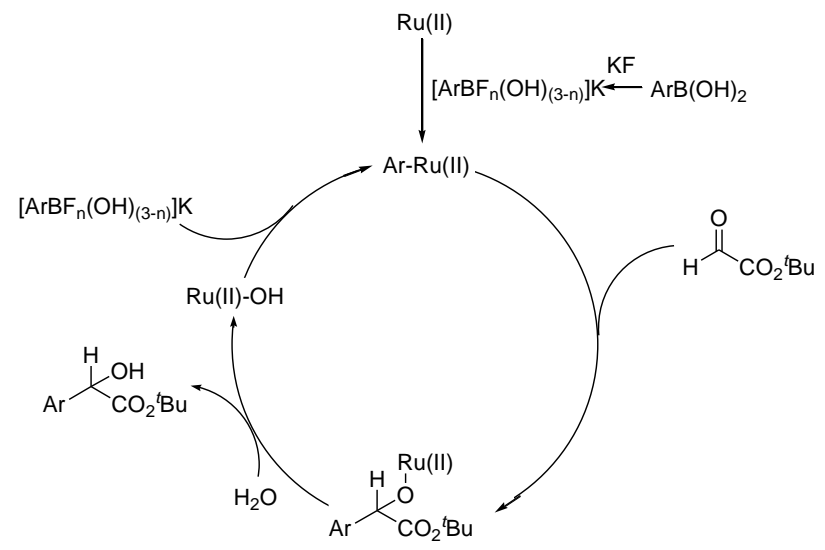

Figure 1 Proposed catalytic cycle

35 To determine the structure of the catalyst, we reacted $\mathrm{RuCl}_{2}\left(\mathrm{PPh}_{3}\right)_{3}$ with $(R, R)$-Me-BIPAM in $\mathrm{CH}_{2} \mathrm{Cl}_{2}$. This provided $\mathrm{RuCl}_{2}\left(\mathrm{PPh}_{3}\right)((R, R)-\mathrm{Me}$-bipam) in $67 \%$ yield (eqn $(1))$. The ${ }^{31} \mathrm{P}$ NMR spectrum of this complex in toluene- $\mathrm{d}_{8}$ showed an ABX pattern. The spectrum is consistent with two cis-phosphorus40 phosphorus interactions and one trans-phosphorus-phosphorus interaction, the trans $J_{p p}$ coupling constant being much greater than the $c i s J_{p p}$ coupling constant (29 ppm $(d d, J=30,495 \mathrm{~Hz})$, $153 \mathrm{ppm}(d d, J=73,495 \mathrm{~Hz}), 170 \mathrm{ppm}(d d, J=30,73 \mathrm{~Hz}))$ (Figure 2). ${ }^{16}$
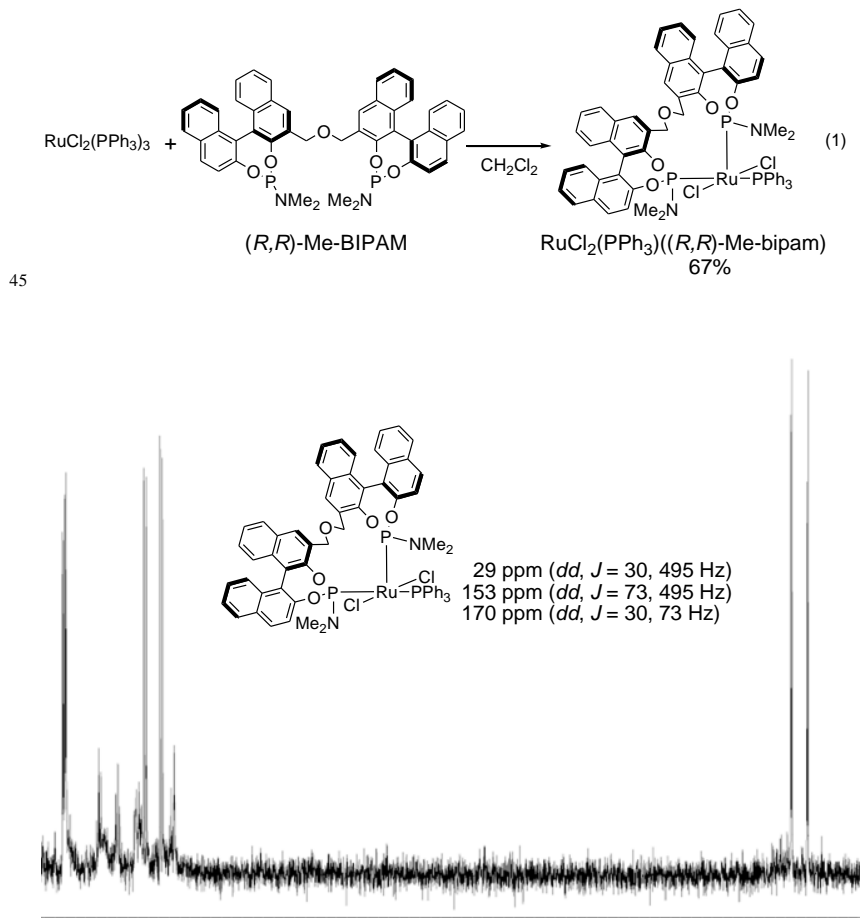

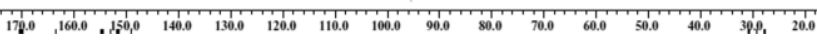

Figure $2{ }^{31} \mathrm{P} \mathrm{NMR}$ of $\mathrm{RuCl}_{2}\left(\mathrm{PPh}_{3}\right)((R, R)-\mathrm{Me}$-bipam $)$ 
The transmetalation between $\mathrm{RuCl}_{2}\left(\mathrm{PPh}_{3}\right)((R, R)$-Me-bipam $)$ and $\operatorname{ArBF}_{n}(\mathrm{OH})_{(3-\mathrm{n})}$ generated by the reaction of $\operatorname{ArB}(\mathrm{OH})_{2}$ and $\mathrm{KF}$ may provide the arylruthenium(II) intermediate $\mathrm{RuCl}(\mathrm{Ar})\left(\mathrm{PPh}_{3}\right)((R, R)-\mathrm{Me}$-bipam), which is analogous to a $\mathrm{Ph}-\mathrm{Cl}$ 5 exchange between $\mathrm{PhB}(\mathrm{OH})_{2}$ and $\left[\mathrm{RuCl}_{2}(p\right.$-cymene $\left.)\left(\mathrm{PPh}_{3}\right)\right]{ }^{17}$ Although isolation of the intermediate $\mathrm{RuCl}(\mathrm{Ph})\left(\mathrm{PPh}_{3}\right)((R, R)-\mathrm{Me}-$ bipam) failed, the reaction of phenylboronic acid and $\mathrm{RuCl}_{2}(p-$ cymene $)\left(\mathrm{PPh}_{3}\right)^{18}$ in the presence of $\mathrm{KF}$ gave $\mathrm{RuCl}(\mathrm{Ph})(p$ cymene) $\left(\mathrm{PPh}_{3}\right)$ in $61 \%$ yield (eqn (2)). ${ }^{17 \mathrm{~b}, 19}$ The enantioselectivity 10 is determined at insertion of the $\mathrm{C}-\mathrm{O}$ double bond into the $\mathrm{C}-\mathrm{Ru}$ bond of $\mathrm{RuCl}(\mathrm{Ar})\left(\mathrm{PPh}_{3}\right)((R, R)-\mathrm{Me}$-bipam) complex.

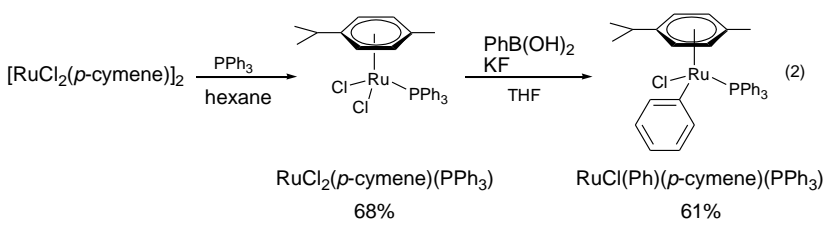

In conclusion, we have developed a $\mathrm{RuCl}_{2}\left(\mathrm{PPh}_{3}\right)_{3} /(R, R)-\mathrm{Me}-$ BIPAM catalyst as an efficient catalytic system for asymmetric 15 addition of arylboronic acids to glyoxylate. With this catalyst system, optically active mandelic acids were easily prepared in up to $99 \%$ ee. To elucidate the enantioselection in the mechanism, characterization of the catalyst and the intermediate is in progress.

This work was supported in part by the Global COE Program 20 (Project No. B01, Catalysis as the Basis for Innovation in Materials Science) from the Ministry of Education, Culture, Sports, Science and Technology, Japan.

\section{Notes and references}

1 (a) Comprehensive Asymmetric Catalysis, ed. E. N. Jacobsen, A.

25 Pfaltz, H. Yamamoto, Springer, Berlin, Germany, 1990; (b) Catalytic Asymmetric Synthesis, ed. I. Ojima, John Wiley \& Sons, Hoboken, New Jersey, 2010.

2 (a) K. Mori, H. Akao, Tetrahedron, 1980, 36, 91-96; (b) M. A. K. Patterson, R. P. Szajewski, G. M. Whitesides, J. Org. Chem., 1981,

30 46, 4682-4685; (c) K. Miyamoto, H. Ohta, J. Am. Chem. Soc., 1990, 112, 4077-4078; (d) S. Tsuchiya, K. Miyamoto, H. Ohta, Biotechnol. Lett., 1992, 14, 1137-1142; (e) D. Basavaiah, P. R. Krishna, Tetrahedron, 1995, 51, 2403-2416; (f) M. M. Kayser, M. D. Mihovilovic, J. Kearns, A. Feicht, J. D. Stewart, J. Org. Chem., 1999, 64, 6603-6608; (g) W. Zhang, P. G. Wang, J. Org. Chem., 2000, 65, 4732-4735; (h) G. DeSantis, Z. Z. William, A. Greenberg, K. Wong, J. Chaplin, S. R. Hanson, B. Farwell, L. W. Nicholson, C. L. Rand, D. P. Winer, D. E. Robertson, M. J. Burk, J. Am. Chem. Soc., 2002, 124, 9024-9025; (i) W. Yang, J.-H. Xu, Y. Xie, Y. Xu, G. Zhao, G.-Q. Lin, Tetrahedron: Asymmetry, 2006, 17, 1769-1774.

3 (a) K. Maruyama, Y. Murakami, K. Yoda, T. Nashino, A. Nishinaga, J. Chem. Soc., Chem. Commun., 1992, 1617-1618; (b) A. E. Russel, S. P. Miller, J. P. Morken, J. Org. Chem., 2000, 65, 8381-8383; (c) K. Ishihara, T. Yano, M. Fushimi, J. Fluorine Chem., 2008, 129, 994997; (d) E. Schmitt, I. Schiffers, C. Bolm, Tetrahedron Lett., 2009, 50, $3185-3188$.

4 (a) H. C. Brown, G. G. Pai, J. Org. Chem., 1985, 50, 1384-1394; (b) J. W. Yang, B. List, Org. Lett., 2006, 8, 5653-5655; (c) J. M. Aizpurua, C. Palomo, R. M. Fratila, P. Ferrón, J. I. Miranda, Tetrahedron, 2010, 66, 3187-3194.

5 (a) J.-F. Carpentier, A. Mortreux, Tetrahedron: Asymmetry, 1997, 8, 1083-1099; (b) F. Cederbaum, C. Lamberth, C. Malan, F. Naud, F. Spindler, M. Studer, H.-U. Blaser, Adv. Synth. Catal., 2004, 346, 842-848; (c) Y. Sun, X. Wan, J. Wang, Q. Meng, H. Zhang, L. Jiang,

55 Z. Zhang, Org. Lett., 2005, 7, 5425-5427; (d) S. Diezi, S. Reimann, N. Bonalumi, T. Mallat, A. Baiker, J. Catal., 2006, 239, 255-262; (e) Q. Meng, Y. Sun, V. Ratovelomanana-Vaidal, J. P. Genêt, Z. Zhang, J.
Org. Chem., 2008, 73, 3842-3847; (c) K. Balázsik, K. Szőri, G. Szőllősi, M. Bartók, Chem. Commun., 2011, 47, 1551-1552.

606 (a) M. Bandini, A. Melloni, A. Umani-Ronchi, Angew. Chem. Int. Ed., 2004, 43, 550-556; (b) T. B. Poulsen, K. A. Jørgensen, Chem. Rev., 2008, 108, 2903-2915; (c) S.-L. You, Q. Cai, M. Zeng, Chem. Soc. Rev., 2009, 38, 2190-2201.

7 (a) N. Gathergood, W. Zhuang, K. A. Jørgensen, J. Am. Chem. Soc., 65 2000, 122, 12517-12522; (b) Y. Yuan, X. Wang, X. Li, K. Ding, J. Org. Chem., 2004, 69, 146-149; (c) S. V. Malhotra, Y. Xiao, Aust. J. Chem., 2006, 59, 468-472; (d) C. Zhu, C. Yuan, Y. Lv, Synlett, 2006, 1221-1224; (e) M. Souedan, J. Collin, R. Gil, Tetrahedron Lett., 2006, 47, 5467-5470; (f) H. Li, Y.-Q. Wang, L. Deng, Org. Lett., 2006, 8, 4063-4065; (g) H.-M. Dong, H.-H. Lu, L.-Q. Lu, C.-B. Chen, W.-J. Xiao, Adv. Synth. Catal., 2007, 349, 1597-1603; (h) J. Kwiatkowski, J. Majer, P. Kwiatknowski, J. Jurczak, Synlett, 2008, 3237-3244; (i) J. Majer, P. Kwiatkowski, J. Jurczak, Org. Lett., 2008, 10, 2955-2958: (j) J. Majer, P. Kwiatkowski, J. Jurczak, Org. Lett., 2009, 11, 46364639; (k) M. Willot, J.-C. Chen, J. Zhu, Synlett, 2009, 577-580.

8 (a) E. J. Ebbers, G. J. A. Ariaans, A. Bruggink, B. Zwanenburg, Tetrahedron: Asymmetry, 1999, 10, 3701-3718; (b) A. Sakakura, S. Uemura, K. Ishihara, Synlett, 2009, 1647-1650; (c) S. K. Alamsetti, G. Sekar, Chem. Commun., 2010, 46, 7235-7237.

${ }_{80} 9$ (a) S. Oi, M. Moro, H. Fukuhara, T. Kawanishi, Y. Inoue, Tetrahedron, 2003, 59, 4351-4361; (b) I. N. Francesco, A. Wagner, F. Colobert, Eur. J. Org. Chem., 2008, 5692-5695; (c) T. Yamamoto, M. Iizuka, H. Takenaka, T. Ohta, Y. Ito, J. Organomet. Chem., 2009, 694, 1325-1332; (d) K. Aikawa, Y. Hioki, K. Mikami, Chem. Asian, 85 J., 2010, 5, 2346-2350; (e) L. Yang, C. A. Correia, C.-J. Li, Adv. Synth. Catal., 2011, 353, 1269-1273.

10 (a) Y. Yamamoto, T. Nishikata, N. Miyaura, J. Synth. Org. Chem., Jpn., 2006, 64, 1112-1121.

11 Y. Yamamoto, T. Nishikata, N. Miyaura, Pure Appl. Chem., 2008, 80, 807-817; (b) N. Miyaura, Synlett, 2009, 2039-2050.

12 (a) Y. Yamamoto, K. Kurihara, N. Miyaura, Angew. Chem. Int. Ed., 2009, 48, 4414-4416; (b) Y. Yamamoto, T. Shirai, M. Watanabe, K. Kurihara, N. Miyaura, Molecules, 2011, 16, 5020-5034.

13 (a) Y. Yamamoto, K. Kurihara, N. Sugishita, K. Oshita, D. Piao, N. Miyaura, Chem. Lett., 2005, 34, 1224-1225; (b) K. Kurihara, N. Sugishita, K. Oshita, D.-G. Piao, Y. Yamamoto, N. Miyaura, J. Organomet. Chem., 2007, 692, 428-435.

14 K. Kurihara, Y. Yamamoto, N. Miyaura, Tetrahedron Lett. 2009, 50, 3158-3160.

10015 (a) K. Kurihara, Y. Yamamoto, N. Miyaura, Adv. Synth. Catal., 2009, 351, 260-270; (b) Y. Yamamoto, Y. Takahashi, K. Kurihara, N. Miyaura, Aust. J. Chem., 2011, 64, 1447-1453.

16 (a) C. W. Jung, P. E. Garrou, P. R. Hoffman, K. G. Caulton, Inorg. Chem., 1984, 23, 726-729; (b) K. S. MacFarlane, A. M. Joshi, S. J. Rettig, B. R. James, Inorg. Chem., 1996, 35, 7304-7310.

17 (a) E. J. Farrington, J. M. Brown, C. F. Barnard, E. Rowsell, Angew. Chem. Int. Ed., 2002, 41, 169-171; (b) E. J. Farrington, C. F. J. Barnard, E. Rowsell, J. M. Brown, Adv. Synth. Catal., 2005, 347, 185-195.

11018 (a) R. A. Zelonka, M. C. Baird, Can. J. Chem., 1972, 50, 3063-3072; (b) E. Hodson, S. J. Simpson, Polyhedron, 2004, 23, 2695-2707.

19 A. S. K. Hashmi, L. Molinari, Organometallics, 2011, 30, 3457-360. 